\title{
Multidimensional Taylor Network Optimal Control of MIMO Nonlinear Systems without Models for Tracking by Output Feedback
}

\author{
Qi-Ming Sun ${ }^{1,2}$ and Hong-Sen Yan ${ }^{1,2}$ \\ ${ }^{1}$ School of Automation, Southeast University, Nanjing 210096, China \\ ${ }^{2}$ Key Laboratory of Measurement and Control of Complex Systems of Engineering, Ministry of Education, Nanjing 210096, China \\ Correspondence should be addressed to Hong-Sen Yan; hsyan@seu.edu.cn
}

Received 15 June 2017; Accepted 14 September 2017; Published 30 October 2017

Academic Editor: Quanxin Zhu

Copyright (C) 2017 Qi-Ming Sun and Hong-Sen Yan. This is an open access article distributed under the Creative Commons Attribution License, which permits unrestricted use, distribution, and reproduction in any medium, provided the original work is properly cited.

\begin{abstract}
The actual controlled objects are generally multi-input and multioutput (MIMO) nonlinear systems with imprecise models or even without models, so it is one of the hot topics in the control theory. Due to the complex internal structure, the general control methods without models tend to be based on neural networks. However, the neuron of neural networks includes the exponential function, which contributes to the complexity of calculation, making the neural network control unable to meet the real-time requirements. The newly developed multidimensional Taylor network (MTN) requires only addition and multiplication, so it is easy to realize real-time control. In the present study, the MTN approach is extended to MIMO nonlinear systems without models to realize adaptive output feedback control. The MTN controller is proposed to guarantee the stability of the closed-loop system. Our experimental results show that the output signals of the system are bounded and the tracking error goes nearly to zero. The MTN optimal controller is proven to promise far better real-time dynamic performance and robustness than the BP neural network self-adaption reconstitution controller.
\end{abstract}

\section{Introduction}

In most input-output control systems, an alteration of one input signal may trigger the change of multiple output signals $[1,2]$. However, in the actual control process, the system's stability is highly demanded [3-5], along with its desirable dynamic performance and multiple tracking with less or even zero errors [6]. Because of the complex internal structure of the multi-input and multioutput (MIMO) system, satisfactory results are hardly gained as a result [7].

Support vector machine stemming from statistical theory is good at handling classification and regression problems. Its great progress has been observed in the fields of nonlinear control [8], fault diagnosis [9], and process modeling [10]. However, at present, it is only applicable to the single variable system due to its complexity.

Recently, great advances for adaptive output feedback control and robust control of nonlinear systems have been achieved [11-13]. Good approximation property of the neural networks provides a new access to adaptive output feedback control [14-16]. For general MIMO nonlinear systems, especially the systems without models, the control methods are always based on neural networks [17-19]. The neuron of neural networks includes the exponential function, which contributes to the complexity of calculation, making the neural network control unable to meet the real-time requirements. Though the larger the number of neural network nodes, the smaller the approximation error, neural network with a large hidden node number tends to complicate the control and the computation of the control system. So, it is difficult to realize the system's real-time control.

Polynomial control method is an intuitive controller design approach. Its design parameters are of clear physical sense [20]. However, it requires an accurate model of the controlled object for solving MIMO nonlinear control problems. 
The multidimensional Taylor network (MTN, whose idea was proposed by Hong-Sen Yan in 2010 and realization was completed by Bo Zhou who is Yan's Ph.D. student) is commonly applied to the analysis of time series prediction [21-28]. MTN, a simple function of the state and input, is good at analyzing and solving the problems in point due to its polynomials. Besides, MTN only involves multiplication and addition, so its simple computation makes desirable real-time control possible. In fact, its computation complexity is nearly equal to that of Taylor expansion of a single neuron of neural network. The idea of MTN optimal control was proposed by Yan [29]. The optimal adjustment controller based on MTN was then developed [30]. However, the parameters of the controller are fixed. The graduate students supervised by Yan have used the simple MTN to solve some practical control engineering problems successfully, such as the plane flight [31], the tank firing control in high speed motion [32], the axisymmetric cruise missile flight for attacking static target [33, 34], and ship roll stabilization [35], all involving MIMO nonlinear systems with strong disturbance. Simulation results show that the simple MTN promises faster response speed, stronger anti-interference capability, and better external stability than PID [31-35], PID neural network $[31,33]$, neural network [34], sliding mode control [31, 34, 35], and active disturbance rejection controller [33].

As has already been pointed out, the existing control methods are known to have some shortcomings, such as needing an accurate model of the controlled object, mainly aiming at the SISO nonlinear system. Some MTN control strategies have also been developed. However, they are just based on the simple MTN (i.e., PID plus the sum of their second-order monomials plus PID, each item of which is multiplied by its corresponding parameter), and the PID parameters are chosen as the initial parameters of MTN controller via the minimum principle, mainly targeting the SISO nonlinear systems. Proposed in the present study is the MIMO feedback control, which, unlike other approaches, does not require the specific format of MTN. Each input of the controlled object corresponds to a subset of internal weights of Multidimensional Taylor Network Controller (MTNC). Even without knowing the internal characteristic parameters of the controlled object, and only by adjusting the internal weights of MTNC, the outputs of the closed-loop system can be made to track the desired signals effectively. Superior to the BP neural network self-adaption reconstitution controller, the MTNC tracks the expectation output curves more satisfactorily as well as suppressing the disturbance more efficiently, promising a better real-time dynamic performance.

The main contributions of this paper are listed as follows:

(1) For the first time, the MTN approach is used to solve the tracking control problem of MIMO nonlinear systems. The computation burden is released due to the simple structure of MTN.

(2) Even with modeling errors, the MTNC proposed in this paper can still be able to satisfy certain performance indexes with excellent dynamic performance.

(3) Compared with the existing control approaches, the proposed control method is simple and accurate.

\section{Problem Statement}

Consider the following MIMO nonlinear system:

$$
\begin{aligned}
\mathbf{x}(k+1) & =\mathbf{f}(\mathbf{x}(k), \mathbf{u}(k)) \\
\mathbf{y}(k) & =\mathbf{h}(\mathbf{x}(k), \mathbf{u}(k)) .
\end{aligned}
$$

After differential homeomorphic transformation, (1) can be converted into (2) [36]. Without losing generality, consider the following MIMO nonlinear system:

$$
\begin{gathered}
x_{r_{1} 1}(k+1)=x_{r_{2} 2}(k) \\
\vdots \\
x_{r_{1}\left(r_{1}-1\right)}(k+1)=x_{r_{1} r_{1}}(k) \\
x_{r_{1} r_{1}}(k+1)=f_{1}(\mathbf{x}(k))+g_{11}(\mathbf{x}(k)) \cdot u_{1}(k)+\cdots \\
+g_{1 m}(\mathbf{x}(k)) \cdot u_{m}(k)+d_{1} \\
\vdots \\
x_{r_{m} 1}(k+1)=x_{r_{m} 2}(k) \quad \vdots \\
x_{r_{m} r_{m}}(k+1)=f_{m}(\mathbf{x}(k))+g_{m 1}(\mathbf{x}(k)) \cdot u_{1}(k)+\cdots \\
+g_{m m}(\mathbf{x}(k)) \cdot u_{m}(k)+d_{m} \\
y_{1}(k)=x_{r_{1} 1}(k) \\
y_{m}(k)=x_{r_{m} 1}(k), \\
\vdots
\end{gathered}
$$

where $\left[r_{1}, r_{2}, \ldots, r_{m}\right]$ is the relative order of the system, and $r_{1}+r_{2}+\cdots+r_{m}=n ; \mathbf{y}=\left[y_{1}, y_{2}, \ldots, y_{m}\right]^{\mathrm{T}} \in R^{m}$ is the output vector of the system; $\mathbf{x}=\left[x_{r_{1} 1}, x_{r_{2} 2}, \ldots, x_{r_{1} r_{1}}\right.$, $\left.\ldots, x_{r_{m} 1}, x_{r_{m} 2}, \ldots, x_{r_{m} r_{m}}\right]^{\mathrm{T}} \in R^{n}$ is the state vector of the system; $\mathbf{u}=\left[u_{1}, u_{2}, \ldots, u_{m}\right]^{\mathrm{T}} \in R^{m}$ is the input vector of the system; $f_{i}(\mathbf{x}(k)), i=1,2, \ldots, m$ is the unknown bounded nonlinear mapping; $g_{i j}(\mathbf{x}(k)), i, j=1,2, \ldots, m$ is the known bounded nonlinear mapping.

Equation (1) can be rewritten as

$$
\begin{aligned}
{\left[\begin{array}{c}
y_{1}\left(k+r_{1}\right) \\
\vdots \\
y_{m}\left(k+r_{m}\right)
\end{array}\right]=} & {\left[\begin{array}{c}
f_{1}(\mathbf{x}(k)) \\
\vdots \\
f_{m}(\mathbf{x}(k))
\end{array}\right]+G(\mathbf{x}(k)) \cdot\left[\begin{array}{c}
u_{1}(k) \\
\vdots \\
u_{m}(k)
\end{array}\right] } \\
& +\left[\begin{array}{c}
d_{1} \\
\vdots \\
d_{m}
\end{array}\right]
\end{aligned}
$$


where $G(\mathbf{x}(k))=\left(\begin{array}{ccc}g_{11}(\mathbf{x}(k)) & \cdots & g_{1 m}(\mathbf{x}(k)) \\ \vdots & \ddots & \vdots \\ g_{m 1}(\mathbf{x}(k)) & \cdots & g_{m m}(\mathbf{x}(k))\end{array}\right)$ is the control gain matrix; $\mathbf{d}=\left[d_{1}, d_{2}, \ldots, d_{m}\right]^{\mathrm{T}}$ is the unknown and bounded disturbance.

From the concept of the dominant input, the $m \times m$ MIMO system can be seen as $m$ multi-input single output system, and the $i$ th subsystem is

$$
\begin{aligned}
y_{i}\left(k+r_{i}\right)= & f_{i}(\mathbf{x}(k))+g_{i 1}(\mathbf{x}(k)) \cdot u_{1}(k)+\cdots \\
& +g_{i i}(\mathbf{x}(k)) \cdot u_{i}(k)+\cdots+g_{i m}(\mathbf{x}(k)) \\
& \cdot u_{m}(k)+d_{i}, \quad i=1,2, \ldots, m .
\end{aligned}
$$

Select a dominant input in the $m$ input, and set $u_{i}(k)$. The rest is treated as interference; then we have

$$
\begin{aligned}
y_{i}\left(k+r_{i}\right)= & f_{i}(\mathbf{x}(k))+g_{i i}(\mathbf{x}(k)) \cdot u_{i}(k)+d_{s i}, \\
& i=1,2, \ldots, m \\
d_{s i}=\sum_{j=1, j \neq i}^{m} g_{i j}(\mathbf{x}(k)) \cdot u_{j}(k)+d_{i} . &
\end{aligned}
$$

Set

$$
\begin{aligned}
A_{i} & =\left[\begin{array}{ccccc}
0 & 1 & 0 & \cdots & 0 \\
0 & 0 & 1 & \cdots & 0 \\
\vdots & \vdots & \vdots & \ddots & 1 \\
0 & 0 & 0 & \cdots & 0
\end{array}\right]_{r_{i} \times r_{i}}, \\
B_{i} & =\left[\begin{array}{llll}
0 & 0 & \cdots & 1
\end{array}\right]_{r_{i} \times 1}^{\mathrm{T}}, \\
C_{i} & =\left[\begin{array}{llll}
1 & 0 & \cdots & 0
\end{array}\right]_{1 \times r_{i}}, \\
\mathbf{x}_{i}(k) & =\left(x_{r_{i 1}}(k), x_{r_{i 2}}(k), \ldots, x_{r_{i} r_{i}}(k)\right)^{\mathrm{T}},
\end{aligned}
$$

and (4) is equivalent to

$$
\begin{aligned}
\mathbf{x}_{i}(k+1)= & A_{i} \cdot \mathbf{x}_{i}(k)+B_{i} \\
& \cdot\left[f_{i}(\mathbf{x}(k))+g_{i i}(\mathbf{x}(k)) \cdot u_{i}(k)+d_{s i}\right] \\
y_{i}\left(k_{i}\right)= & C_{i} \cdot \mathbf{x}(k), \quad i=1,2, \ldots, m .
\end{aligned}
$$

Assuming only output can be measured, and

$$
\begin{aligned}
g_{i i}(\mathbf{x}(k)) & \geq g_{0}>0, \\
\left|d_{s i}\right| & \leq D, \quad \text { where } D \text { is an unknown constant, }
\end{aligned}
$$

the expected output of the system is

$$
\mathbf{y}_{\mathrm{d}}(k)=\left[y_{\mathrm{d} 1}(k), y_{\mathrm{d} 2}(k), \ldots, y_{\mathrm{d} m}(k)\right]^{\mathrm{T}},
$$

the desired state vector of the $i$ th subsystem is

$$
\mathbf{x}_{\mathrm{d} i}(k)=\left[y_{\mathrm{d} i}(k), y_{\mathrm{d} i}(k+1), \ldots, y_{\mathrm{d} i}\left(k+r_{i}-1\right)\right]^{\mathrm{T}},
$$

and the tracking error is

$$
e_{i}(k)=y_{\mathrm{d} i}(k)-y_{i}(k) .
$$

To prove that the tracking error converges to 0 , set the tracking error vector

$$
\mathbf{e}_{i}(k)=\mathbf{x}_{\mathrm{d} i}(k)-\mathbf{x}_{i}(k) .
$$

Control target: for the affine nonlinear MIMO systems, without state observation, design the adaptive MTN optimal output feedback controller to make the nonlinear system (2) stable, enabling its output to track the desired signal and its parameters and tracking error to be uniformly bounded.

Define the filtering error as

$$
\begin{aligned}
s_{i}(k)=\Lambda \cdot \mathbf{e}_{i}(k)=[\tau, \tau, \ldots, \tau] \cdot \mathbf{e}_{i}(k), & \\
& i=1,2, \ldots m,
\end{aligned}
$$

where $\Lambda=[\tau, \tau, \ldots, \tau], \tau>0$ is the design constant.

Theorem 1. Assuming system (5) with $f_{i}(\mathbf{x}(k))$ and $g_{i i}(\mathbf{x}(k))$ is known and $d_{s i}=0$, the tracking error then converges to 0 if the optimal control input is set as

$$
u_{i}^{*}(k)=T s(k) \cdot e_{i}(k)+u_{i n}^{*}(k)
$$

where $u_{\text {in }}^{*}(k)=\left(y_{d i}\left(k+r_{i}\right)-f_{i}(\mathbf{x}(k))-e_{i}(k)\right) / g_{i i}(\mathbf{x}(k)), T s(k)$ is the design constant, and $T s(k) \cdot e_{i}(k) \cdot g_{i i}(\mathbf{x}(k))>0$.

Proof. Taking the Lyapunov function candidate as

$$
s_{i}(k)=\Lambda \cdot \mathbf{e}_{i}(k)=[\tau, \tau, \ldots, \tau] \cdot \mathbf{e}_{i}(k),
$$

we have

$$
\begin{aligned}
\Delta s_{i}(k)= & s_{i}(k+1)-s_{i}(k)=\Lambda \cdot \mathbf{e}_{i}(k+1)-\Lambda \cdot \mathbf{e}_{i}(k) \\
= & y_{\mathrm{d} i}\left(k+r_{i}\right)-e_{i}(k)-y_{i}\left(k+r_{i}\right) \\
= & y_{\mathrm{d} i}\left(k+r_{i}\right)-e_{i}(k) \\
& -\left[f_{i}(\mathbf{x}(k))+g_{i i}(\mathbf{x}(k)) \cdot u_{i}(k)+d_{s i}\right] .
\end{aligned}
$$

Substituting $u_{i}^{*}(k)$ and $d_{s i}=0$ into (7) yields

$$
\Delta s_{i}(k)=-T s(k) \cdot e_{i}(k) \cdot g_{i i}(\mathbf{x}(k))<0 .
$$

According to the Lyapunov theorem, this result means $\lim _{k \rightarrow \infty} s_{i}(k)=0$.

That completes the proof.

However, $d_{s i} \neq 0$. So other methods are needed to suppress the interference. As $f_{i}(\mathbf{x}(k))$ is unknown, $u_{i n}^{*}(k)$ is unobtainable. Thus, the MTN is used to approximate $u_{i n}^{*}(k)$, and $u_{s i}(k)$ is added to interference suppression.

\section{Multidimensional Taylor Network}

MTN can approximate any nonlinear functions with the finite point of discontinuity. Compared with the existing methods based on neural networks, MTN has the following merits: 
(1) being neatly structured; (2) being good at representing or approximating to nonlinear dynamical systems; (3) guaranteeing real-time control by only addition or multiplication operations allowable. In addition, as with the neural networks, only the internal weights of MTNC are required to be adjusted to make the outputs of the closed-loop system track the desired signals effectively.

By the function approximation of MTN, we obtain

$$
\begin{aligned}
u_{i n}^{*}(k) & =\frac{\left(y_{\mathrm{d} i}\left(k+r_{i}\right)-f_{i}(\mathbf{x}(k))-e_{i}(k)\right)}{g_{i i}(\mathbf{x}(k))} \\
& =W_{i}^{*}(k) \cdot \Phi(\boldsymbol{\eta}(k))+\varepsilon,
\end{aligned}
$$

where approximation error $\varepsilon$ satisfies the following conditions:

$$
|\varepsilon| \leq \varepsilon_{0}
$$

where $\varepsilon_{0}$ is the given normal number, and

$$
\begin{aligned}
& \boldsymbol{\eta}(k) \\
& \quad=\left[e_{1}(k), e_{1}(k-1), \ldots, e_{i}(k), \ldots, e_{m}(k), \ldots\right]^{\mathrm{T}} .
\end{aligned}
$$

For the convenience of writing, denote the number of elements of $\boldsymbol{\eta}(k)$ as $n_{z}$, and we have

$$
\begin{aligned}
\mathbf{z}(k) & =\left[z_{1}(k), z_{2}(k), \ldots, z_{n_{z}}(k)\right]^{\mathrm{T}} \\
& =\left[e_{1}(k), e_{1}(k-1), \ldots, e_{i}(k), \ldots, e_{m}(k), \ldots\right]^{\mathrm{T}} .
\end{aligned}
$$

The basic structure of MTN is shown in Figure 1. The output of MTN $u_{i n}(k)$ can be expressed as

$$
u_{\text {in }}(k)=\sum_{j=1}^{N\left(n_{z}, t\right)} w_{i j}(k) \prod_{s=1}^{n} z_{i}^{\lambda_{s, j}}(k) \text {, }
$$

where $N\left(n_{z}, t\right)$ is the total number of the expansions, $w_{i j}(k)$ is the weight of the $j$ th product term, $\lambda_{(s, j)}$ is the power of $z_{s}(k)$ in the $j$ th product term, and $\sum_{s=1}^{n} \lambda_{s, j} \leq t$.

Setting $\Phi(\boldsymbol{\eta}(k))=\left[1, z_{1}(k), z_{2}(k), \ldots, z_{n_{z}}(k), \ldots, z_{1}^{2}(k)\right.$, $\left.z_{1}(k) z_{2}(k), \ldots, z_{n_{z}}^{t}(k)\right]^{\mathrm{T}}$ gives

$$
u_{i n}^{*}(k)=W_{i}^{*}(k) \cdot \Phi(\boldsymbol{\eta}(k))+\varepsilon .
$$

The optimal parameter $W_{i}^{*}(k)$ is defined as

$$
\begin{aligned}
W_{i}^{*} & (k) \\
& =\arg \min _{W_{i} \in \Omega_{w}}\left\{\sup \left|W_{i}(k) \cdot \Phi(\boldsymbol{\eta}(k))-u_{i n}^{*}(k)\right|\right\},
\end{aligned}
$$

where $\Omega_{w}=\left\{W_{i} \mid\left\|W_{i}\right\| \leq w_{m}\right\}, w_{m}>0$ is the design constant.

Then the MTN output feedback controller can be written as

$$
\begin{aligned}
u_{i}^{*}(k)=\operatorname{Ts}(k) \cdot e_{i}(k)+\widehat{u}_{i n}(k)+u_{s i}(k), & \\
& i=1,2, \ldots, m,
\end{aligned}
$$

where $\widehat{u}_{i n}(k)=\widehat{W}_{i}(k) \cdot \Phi(\boldsymbol{\eta}(k))$ is the output of the MTN, $\widehat{W}_{i}(k)$ is the estimated value of the optimal weight $W_{i}^{*}(k)$, and $u_{s i}$ is the robust control term:

$$
u_{s i}(k)=\frac{D+g_{0} \cdot \varepsilon_{0}}{g_{0}} \cdot \operatorname{sgn}\left(s_{i}(k)\right) .
$$

The control structure is shown in Figure 2.

\section{Parameter Adjustment}

To ensure that the output $y_{i}(k)$ of the controlled object follows the desired input $r_{i}(k)$, the parameters $\mathbf{W}_{i}(k)$ of MTN controller are adjusted by back-propagation learning algorithm.

Specific adjustments go as follows:

$$
\text { Set } E=\frac{1}{l} \sum_{i=1}^{m} \sum_{k=1}^{l}\left[r_{i}(k)-y_{i}(k)\right]^{2}=\frac{1}{l} \sum_{i=1}^{m} \sum_{k=1}^{l} e_{i}^{2}(k) .
$$

And we get

$$
w_{i j}(k+1)=w_{i j}(k)-\alpha_{j}(k) \frac{\partial E}{w_{i j}(k)},
$$

where

$$
\begin{aligned}
\frac{\partial E}{w_{i j}(k)} & =\sum_{i=1}^{m} \frac{\partial E}{\partial y_{i}(k)} \cdot \frac{\partial y_{i}(k)}{\partial w_{i j}(k)} \\
\frac{\partial y_{i}(k)}{\partial w_{i j}(k)} & =\frac{\partial y_{i}(k)}{\partial u_{i}(k)} \cdot \frac{\partial u_{i}(k)}{\partial w_{i j}(k)} .
\end{aligned}
$$

The system structure is unknown. However, the influence of the real system by output $u_{i}(k)$ of controller can be obtained, so $\partial y_{i}(k) / \partial u_{i}(k)$ can be replaced by the sign function

$$
\operatorname{sgn}\left[\frac{y_{i}(k+1)-y_{i}(k)}{u_{i}(k)-u_{i}(k-1)}\right] .
$$

This alternative is feasible, as the numerical size of $\partial y_{i}(k) /$ $\partial u_{i}(k)$, as an intermediate product term, can be adjusted by step $\alpha_{j}(k)$. But it does not change the direction of the adjustment. Simultaneously, it can prevent the numerical size from going too large by the two-step output of the MTN convergence.

Step $\alpha_{j}(k)$ uses the following adaptive adjustment strategy:

$$
\alpha_{j}(0)=\alpha_{j 0},
$$

where $\alpha_{j 0}$ is a given constant as the initial value,

$$
\alpha_{j}(k+1)= \begin{cases}\beta \cdot \alpha_{j}(k) & \left\|\frac{\partial E}{w_{i j}(k)}\right\|<\left\|\frac{\partial E}{w_{i j}(k+1)}\right\| \\ \gamma \cdot \alpha_{j}(k) & \left\|\frac{\partial E}{w_{i j}(k)}\right\| \geq\left\|\frac{\partial E}{w_{i j}(k+1)}\right\|,\end{cases}
$$

where $\beta>1,0<\gamma<1$, and $\left\|\partial E / w_{i j}(k)\right\|$ is the norm of $\partial E / w_{i j}(k)$. 


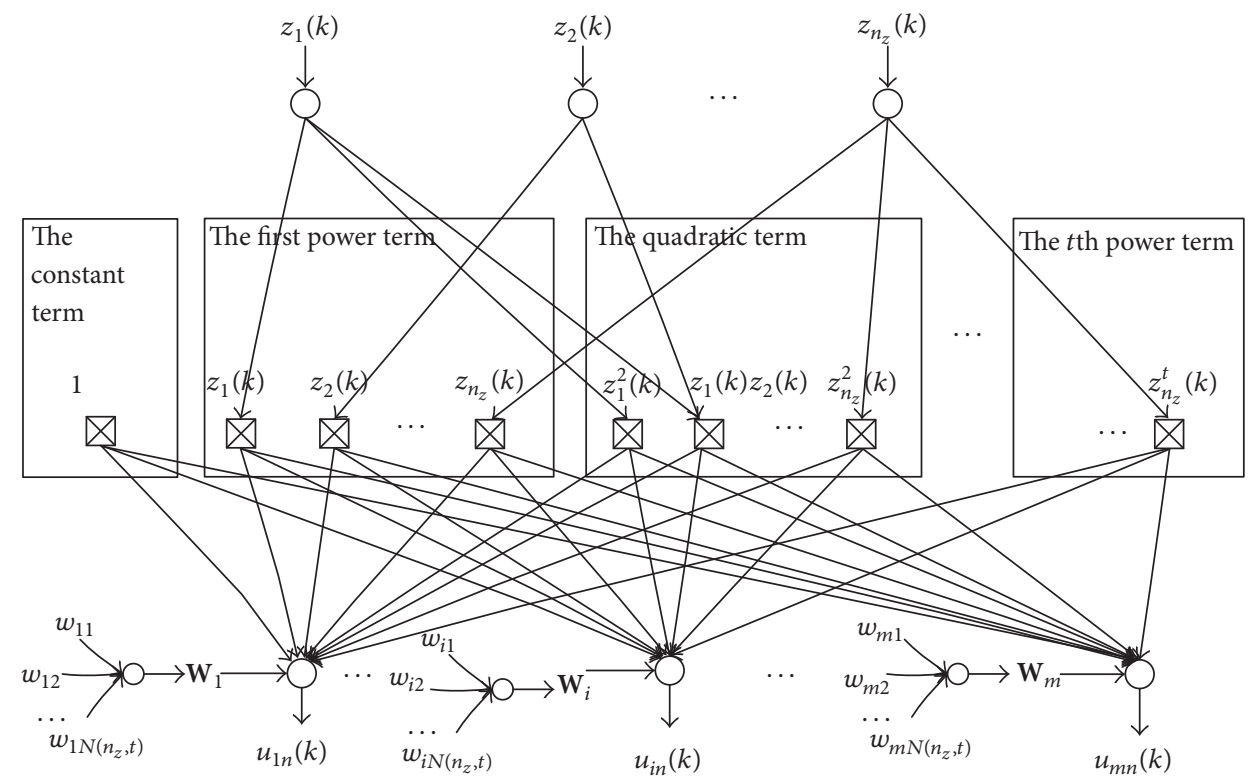

Figure 1: The basic structure of MTN.

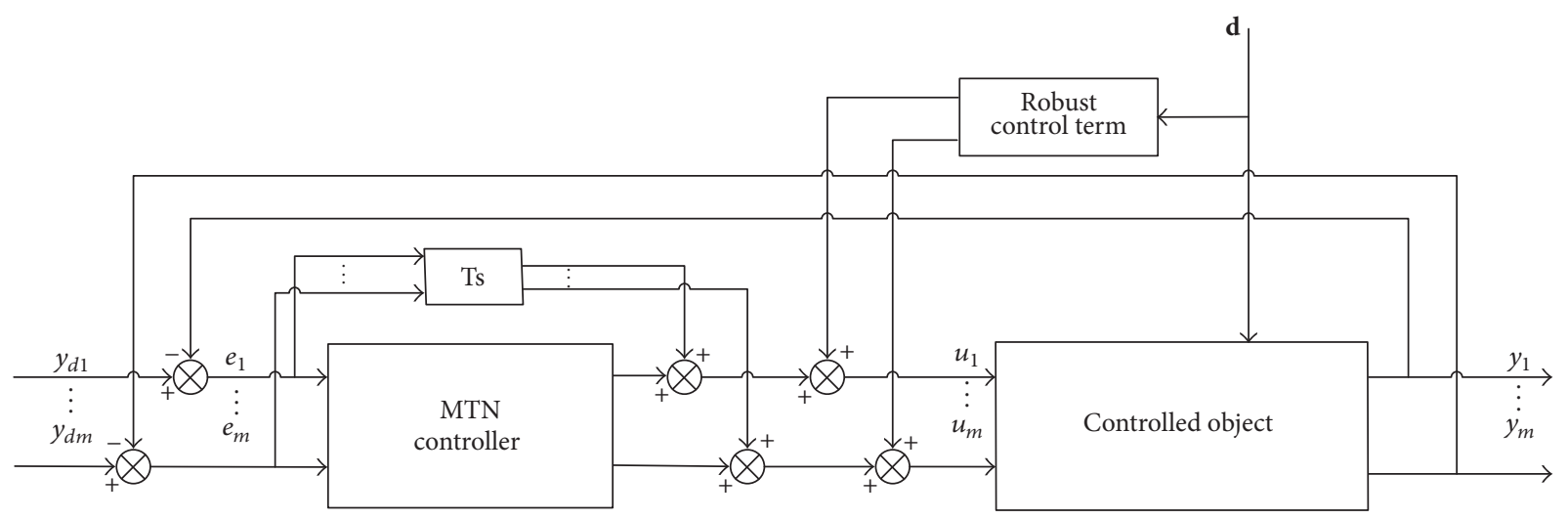

FIgURE 2: The control structure.

\section{Simulation Results}

For demonstration of the effectiveness of the proposed method, consider the following nonlinear system. No specific parameters of the controlled object are needed in the control process.

$$
\begin{aligned}
& x_{1}(k+1)=x_{1}(k)+\mathrm{Ts} \cdot\left(x_{2}(k)-2.6 \cdot x_{1}(k)\right) \\
& x_{2}(k+1)=x_{2}(k)+\mathrm{Ts} \cdot\left(-0.68 \cdot x_{2}(k)-0.8 \cdot \pi\right. \\
& \cdot x_{1}(k) \cdot x_{2}(k)-0.16 \cdot \pi \cdot x_{1}^{3}(k)+u_{1}(k)+0.2 \\
& \left.\cdot x_{3}(k) \cdot u_{2}(k)\right) \\
& x_{3}(k+1)=x_{3}(k)+\mathrm{Ts} \cdot\left(x_{4}(k)-3.2 \cdot x_{3}(k)\right)
\end{aligned}
$$

$$
\begin{aligned}
& x_{4}(k+1)=x_{4}(k)+\mathrm{Ts} \cdot\left(-0.37 \cdot \pi \cdot x_{4}(k)-2.4\right. \\
& \quad x_{4}(k) \cdot x_{3}(k)-0.5 \cdot x_{3}^{3}(k)+0.2 \cdot \frac{1-e^{-x_{1}(k)}}{1+e^{-x_{1}(k)}} \\
& \left.\quad+u_{2}(k)+0.3 \cdot u_{1}(k)\right) \\
& y_{1}(k)=x_{1}(k) \\
& y_{2}(k)=x_{3}(k),
\end{aligned}
$$

where Ts $=0.1$. 

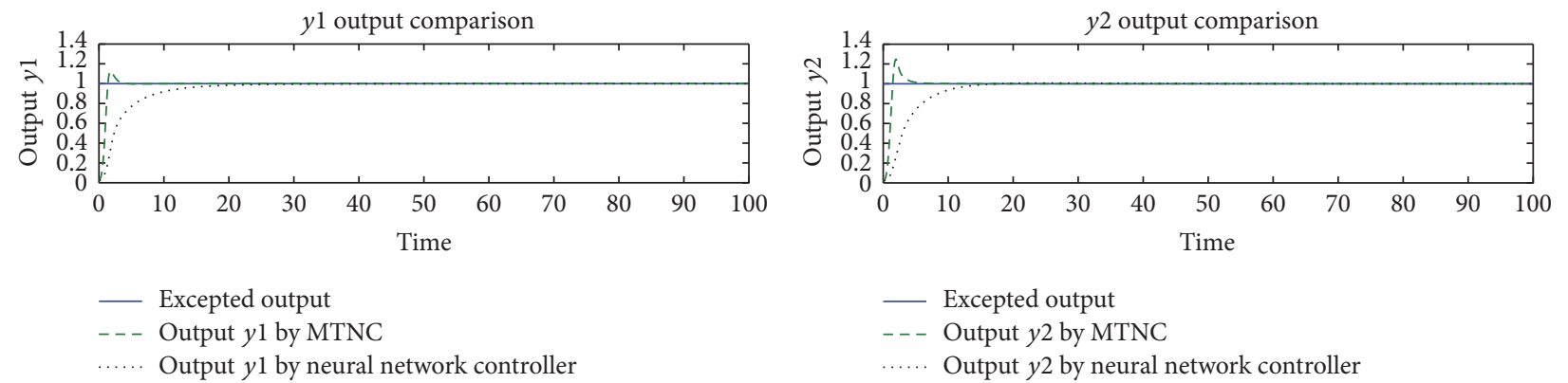

FIgURE 3: Output comparison.
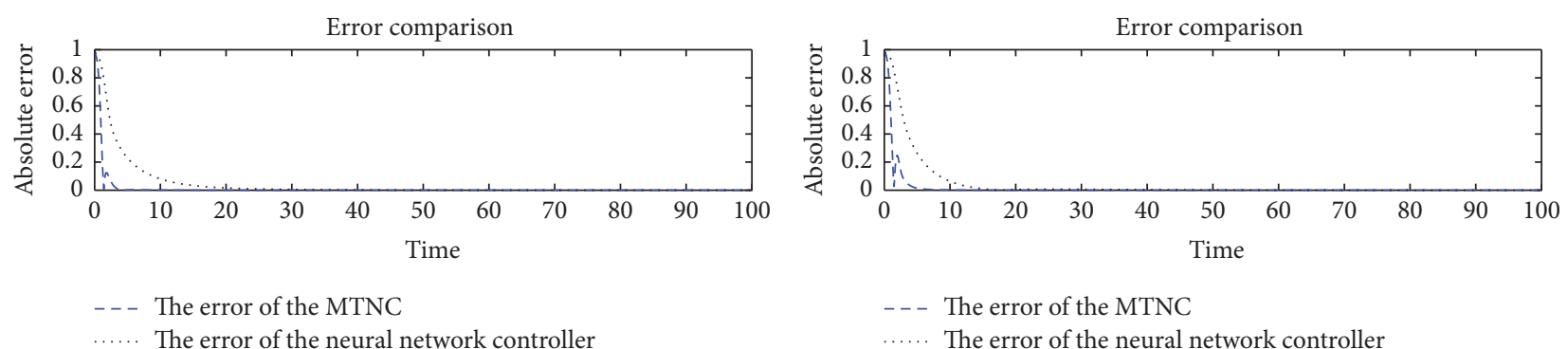

Figure 4: Absolute error curve.

The initial parameters of the system are

$$
\begin{aligned}
& x_{1}(0)=0.005, \\
& x_{2}(0)=0.15, \\
& x_{3}(0)=0.005, \\
& x_{4}(0)=0.15 .
\end{aligned}
$$

5.1. Step Response. For the proposed MTNC, when the dimension $n$ of the controller is equal to 2, the unit step response curve is shown in Figure 3.

The unit step response curve of the neural network selfadaption reconstitution controller is presented in Figure 3, which reveals that the BP neural network self-adaption reconstitution controller responds slowly, while the MTNC has a much faster response speed. Figure 4 illustrates the absolute tracking error curve.

5.2. Antijamming Capability. For demonstration of the antijamming capability of the controller, when $k$ is greater than 60 , a sinusoidal signal with an amplitude of 0.12 is superimposed on $x_{2}$ and $x_{4}$, respectively, and the response curve is shown in Figure 5.

From Figure 5, it can be seen that the MTNC is of higher antijamming capability.

The absolute error curve is displayed in Figure 6.

5.3. Tracking Ability. When $k>10$, with the expected output curves $y_{d 1}$ and $y_{d 2}$ overlaying the cosine signal with an amplitude of 0.1 to verify the follow-up response performance of the controller, the response curves are shown in Figure 7.
As indicated by Figure 7, the proposed design approach tracks the desired signal more quickly.

5.4. Robustness. The amplitude of disturbance is expanded 5 times to verify the robustness of the controller, and the response curve is given in Figure 8.

Experimental results demonstrate that the MTNC based on the multidimensional Taylor network is of better robustness for disturbance.

5.5. Summary. In short, by realizing the optimal closed-loop tracking control of the MIMO nonlinear systems through output feedback with no model involved, the MTNC guarantees its faster response speed, robustness, and antidisturbance capability.

Besides, the MTNC, with good real-time capability and low computational complexity, does not contain the exponential function, so it is easy to achieve real-time control. Taking ten nodes as an example and supposing that the highest order of the exponential function expansion of the neural network is three, the controller of the neural network needs 98 additions and 160 multiplications, while the MTNC needs only 18 additions and 30 multiplications. Therefore, the reduction of calculation complexity greatly improves the realtime performance of the proposed controller.

\section{Conclusions}

The MIMO nonlinear system control method based on the multidimensional Taylor network has been proposed here. Compared with the traditional methods, MTN, as a kind of dynamic model, can be seen as a system model. In the control 

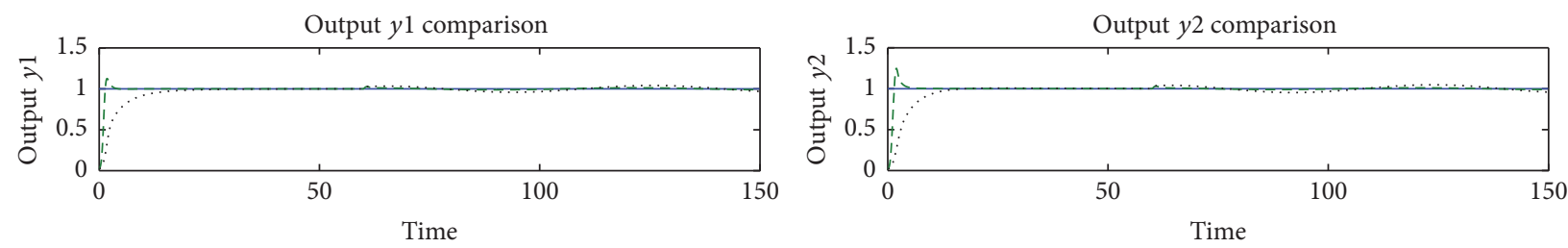

_ Excepted output

- - - Output $y 1$ by MTNC

..... Output $y 1$ by neural network controller

_ Excepted output

- - - Output $y 1$ by MTNC

..... Output $y 2$ by neural network controller

FIGURE 5: Output comparison.
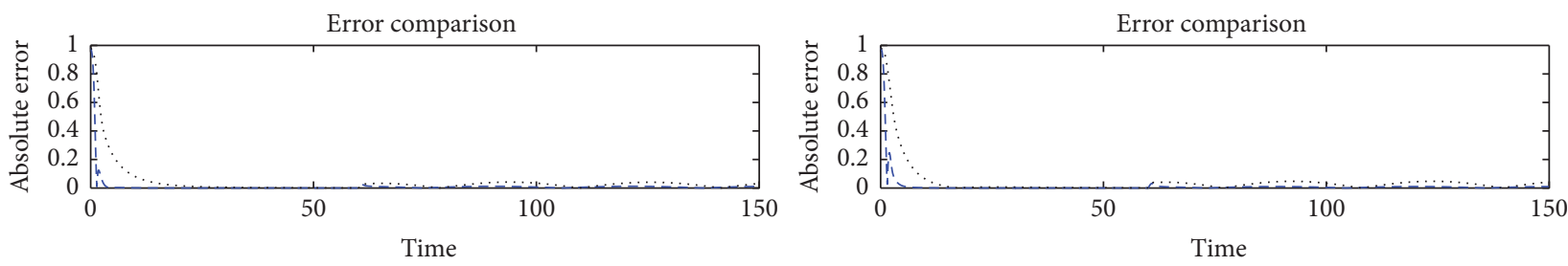

- . - The error of the MTNC

- . - The error of the MTNC

..... The error of the neural network controller

..... The error of the neural network controller

FIgURE 6: Absolute error curve.
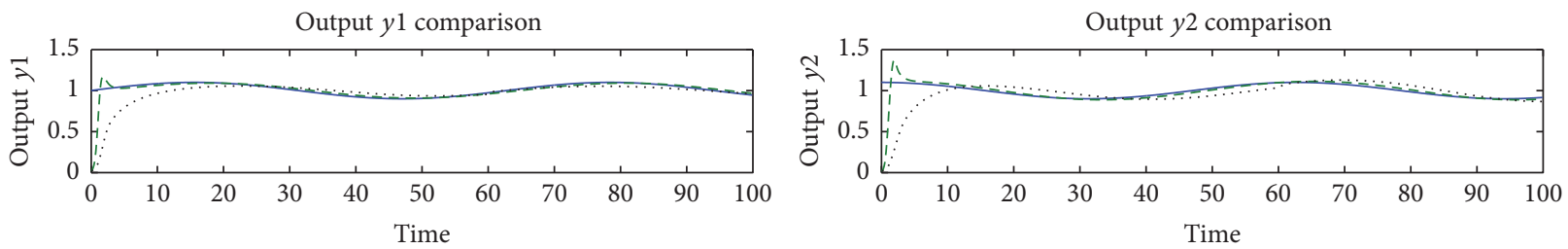

- Excepted output

- - - Output $y 1$ by MTNC

..... Output $y 1$ by neural network controller

- Excepted output

- - - Output $y 2$ by MTNC

...... Output $y 2$ by neural network controller

FIGURE 7: Output comparison.

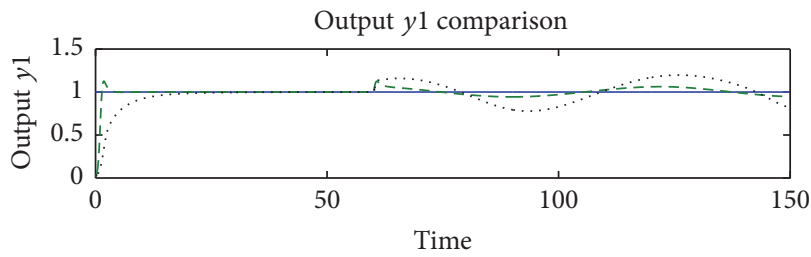

Excepted output

- - Output $y 1$ by MTNC

..... Output $y 1$ by neural network controller

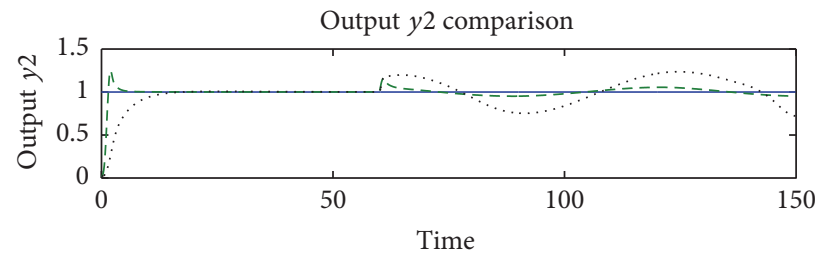

- Excepted output

- - - Output $y 2$ by MTNC

..... Output $y 2$ by neural network controller

FIGURE 8: Output comparison.

process, the closed-loop tracking control of nonlinear system was realized by output feedback without state observation. Due to the Lyapunov function, the tracking error turned out to be nearly zero. The MTNC parameter adjustment algorithm without the specific parameters of the controlled object was developed as well.
During the experiments, the MTNC tracked the expected output curves satisfactorily, capable of suppressing the disturbance efficiently when a sinusoidal disturbance was superimposed on $x_{2}$ and $x_{4}$; the real-time dynamic performance of the controller has been significantly improved due to the reduction of calculation complexity. 


\section{Conflicts of Interest}

The authors declare that they have no conflicts of interest.

\section{Authors' Contributions}

Qi-Ming Sun and Hong-Sen Yan contributed equally to this work.

\section{Acknowledgments}

This work was supported in part by the National Natural Science Foundation of China under Grants 61673112 and 60934008, the Fundamental Research Funds for the Key Universities of China under Grants 2242017K10003 and 2242014K10031, and the Priority Academic Program Development of Jiangsu Higher Education Institutions (PAPD). The authors thank Professor Li Lu for valuable comments and suggestions.

\section{References}

[1] L. Mo and M. M. Bayoumi, "Adaptive pole placement control of MIMO systems," Institute of Electrical and Electronics Engineers Transactions on Automatic Control, vol. 38, no. 6, pp. 967-970, 1993.

[2] S. S. Xiong and Q. X. Zhu, "Decentralized risk-sensitive design for large-scale stochastic interconnected systems with timevarying delays," Journal of The Franklin Institute, vol. 353, no. 7, pp. 1527-1552, 2016.

[3] G. Pin and T. Parisini, "Networked predictive control of uncertain constrained nonlinear systems: recursive feasibility and input-to-state stability analysis," Institute of Electrical and Electronics Engineers Transactions on Automatic Control, vol. 56, no. 1, pp. 72-87, 2011.

[4] B. Wang and Q. X. Zhu, "Stability analysis of Markov switched stochastic differential equations with both stable and unstable subsystems," Systems \& Control Letters, vol. 105, pp. 55-61, 2017.

[5] Q. X. Zhu and Q. Y. Zhang, "Pth moment exponential stabilisation of hybrid stochastic differential equations by feedback controls based on discrete-time state observations with a time delay," IET Control Theory Applications, vol. 11, no. 12, pp. 19922003, 2017.

[6] A. Mannava, S. N. Balakrishnan, L. Tang, and R. G. Landers, "Optimal tracking control of motion systems," IEEE Transactions on Control Systems Technology, vol. 20, no. 6, pp. 15481558, 2012.

[7] Q. X. Zhu, "Razumikhin-type theorem for stochastic functional differential equations with Lévy noise and Markov switching," International Journal of Control, vol. 90, no. 8, pp. 1703-1712, 2017.

[8] C. J. Ong, D. Sui, and E. G. Gilbert, "Enlarging the terminal region of nonlinear model predictive control using the support vector machine method," Automatica, vol. 42, no. 6, pp. 10111016, 2006.

[9] H. Yi, X. F. Song, B. Jiang, and D. C. Wang, "Support vector machine based on nodes refined decision directed acyclic graph and its application to fault diagnosis," Acta Automatica Sinica, vol. 36, no. 3, pp. 427-432, 2010.

[10] I. Goethals, K. Pelckmans, J. A. K. Suykens, and B. D. Moor, "Identification of MIMO Hammerstein models using least squares support vector machines," Automatica, vol. 41, no. 7, pp. 1263-1272, 2005.

[11] J. X. Li, C. Y. Gao, Y. M. Fang, C. Guo, and W. B. Zhang, "Adaptive regulation of a class of uncertain nonlinear systems with nonstrict feedback form," Mathematical Problems in Engineering, Article ID 9748489, pp. 1-7, 2016.

[12] N. Hovakimyan, B. J. Yang, and A. J. Calise, "Adaptive output feedback control methodology applicable to non-minimum phase nonlinear systems," Automatica, vol. 42, no. 4, pp. 513$522,2006$.

[13] H. Wang and Q. X. Zhu, "Finite-time stabilization of highorder stochastic nonlinear systems in strict-feedback form," Automatica, vol. 54, pp. 284-291, 2015.

[14] J. Vance and S. Jagannathan, "Discrete-time neural network output feedback control of nonlinear discrete-time systems in non-strict form," Automatica, vol. 44, no. 4, pp. 1020-1027, 2008.

[15] Z. Wu, J. Lu, J. Shi, Y. Liu, and Q. Zhou, "Robust adaptive neural control of morphing aircraft with prescribed performance," Mathematical Problems in Engineering, pp. 1-16, 2017.

[16] Q. X. Zhu and J. D. Cao, "Exponential stability of stochastic neural networks with both Markovian jump parameters and mixed time delays," IEEE Transactions on Systems, Man, and Cybernetics, Part B: Cybernetics, vol. 41, no. 2, pp. 341-353, 2011.

[17] Q. Yang, Z. Yang, and Y. Sun, "Universal neural network control of MIMO uncertain nonlinear systems," IEEE Transactions on Neural Networks and Learning Systems, vol. 23, no. 7, pp. 1163$1169,2012$.

[18] Q. X. Zhu and J. D. Cao, "Stability analysis of markovian jump stochastic BAM neural networks with impulse control and mixed time delays," IEEE Transactions on Neural Networks and Learning Systems, vol. 23, no. 3, pp. 467-479, 2012.

[19] Q. X. Zhu, J. D. Cao, and R. Rakkiyappan, "Exponential input-to-state stability of stochastic Cohen-Grossberg neural networks with mixed delays," Nonlinear Dynamics, vol. 79, no. 2, pp. 1085-1098, 2015.

[20] C. Samia and S. Lassaad, "Polynomial approach to nonlinearsystem control via the exact state-input linearization method," in Proceedings of the 16th International Conference on Sciences and Techniques of Automatic Control and Computer Engineering, STA 2015, pp. 905-911, tun, December 2015.

[21] Y. Lin, H. S. Yan, and B. Zhou, "Nonlinear time series prediction method based on multi-dimensional Taylor network and its applications," Control and Decision, vol. 29, no. 5, pp. 795-801, 2014, (in Chinese).

[22] Y. Lin, H. S. Yan, and B. Zhou, "A novel modeling method based on multi-dimensional Taylor network and its application in time series prediction," Advanced Materials Research, vol. 940, pp. 480-484, 2014.

[23] Y. Lin, H. S. Yan, and B. Zhou, "A novel nonlinear time series prediction method and its application in structural vibration response prediction," Applied Mechanics and Materials, vol. 599601, pp. 1918-1921, 2014.

[24] B. Zhou and H. S. Yan, "Financial time series forecasting based on wavelet and multi-dimensional Taylor network dynamics model," Systems Engineering-Theory and Practice, vol. 33, no. 10, pp. 2654-2662, 2013, (in Chinese).

[25] B. Zhou and H. S. Yan, "Time series forecasting based on the empirical mode decomposition multi-dimensional Taylor network model," in Proceedings of the 2013 9th International Conference on Natural Computation, ICNC 2013, pp. 1194-1198, chn, July 2013. 
[26] B. Zhou and H. S. Yan, "Nonlinear system identification and prediction based on dynamics cluster multi-dimensional Taylor network model," Control and Decision, vol. 29, no. 1, pp. 33-38, 2014, (in Chinese).

[27] B. Zhou and H. S. Yan, "A dynamics model based on intermittent feedback multi-dimensional Taylor network," ACTA Automatica Sinica, vol. 40, no. 7, pp. 1517-1521, 2014, (in Chinese).

[28] H. S. Yan and A. M. Kang, "Asymptotic tracking and dynamic regulation of SISO nonlinear system based on discrete multidimensional Taylor network," in IET Control Theory and Applications, pp. 10-1049, 2017.

[29] H. S. Yan, "MTN (multi-dimensional Taylor network) optimal control," Tech. Rep., Research Institute of Control and Optimization of Manufacturing Systems, School of Automation, Southeast University, Nanjing, China, 2010, (in Chinese).

[30] Q. M. Sun and H. S. Yan, "Optimal adjustment control of SISO nonlinear systems based on multi-dimensional taylor network only by output feedback," Advanced Materials Research, vol. 1049-1050, pp. 1389-1391, 2014.

[31] Y. Zhou, Research and simulation of multi-dimensional Taylor network optimal control for the flight of the plane [M.S. thesis], Southeast University, Dhaka, Bangladesh, 2016, (in Chinese).

[32] T. Jin, Research and simulation of multi-dimensional Taylor network optimal control for the tank firing control in high speed motion [M.S. thesis], Southeast University, Dhaka, Bangladesh, 2016, (in Chinese).

[33] L. C. Xia, Research into multi-dimensional Taylor network optimal control of the axisymmetric cruise missile flight for attacking static target [M.S. thesis], Southeast University, Dhaka, Bangladesh, 2016, (in Chinese).

[34] J. X. Zhang, Research and simulation of the cruise missile flight trajectory control [M.S. thesis], Southeast University, Dhaka, Bangladesh, 2015, (in Chinese).

[35] Y. Yang, Study on multi-dimensional Taylor network optimal control for ship roll stabilization [M.S. thesis], Southeast University, Dhaka, Bangladesh, 2016, (in Chinese).

[36] G. Bartolini and E. Punta, "Reduced-order observer in the sliding-mode control of nonlinear nonaffine systems," Institute of Electrical and Electronics Engineers Transactions on Automatic Control, vol. 55, no. 10, pp. 2368-2373, 2010. 


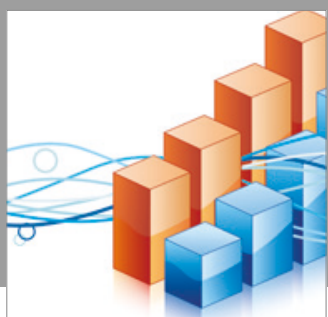

Advances in

Operations Research

vatersals

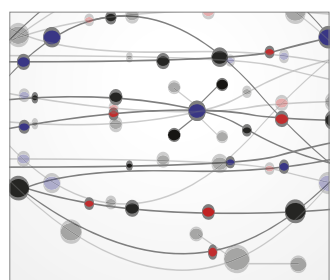

\section{The Scientific} World Journal
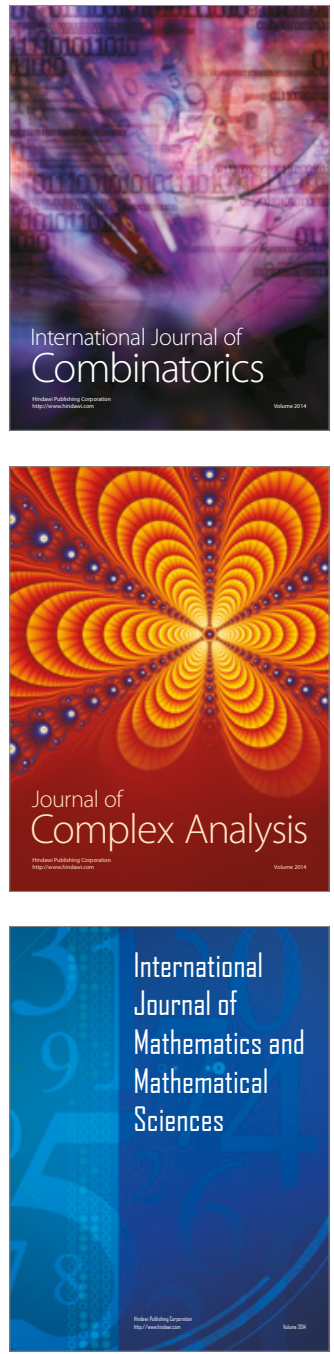
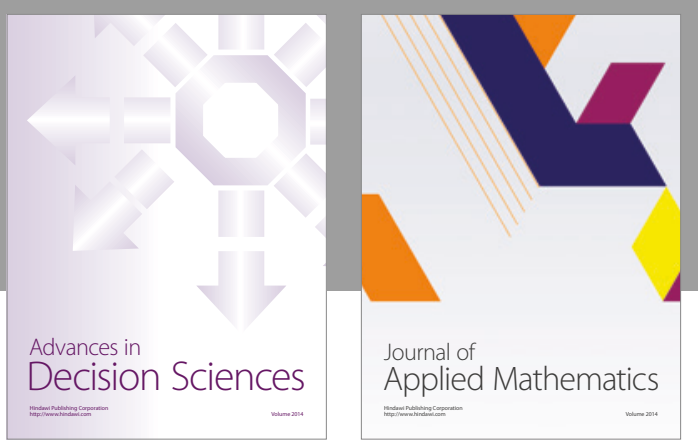

Algebra

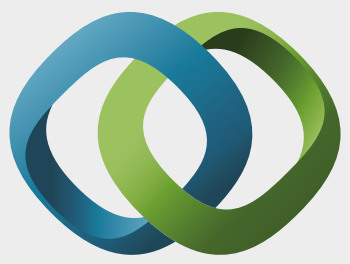

\section{Hindawi}

Submit your manuscripts at

https://www.hindawi.com
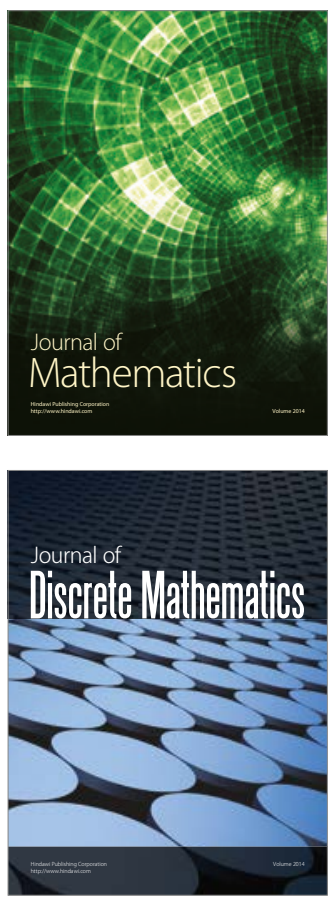

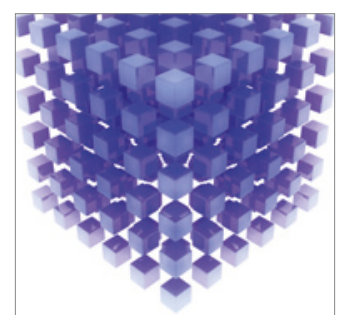

Mathematical Problems in Engineering
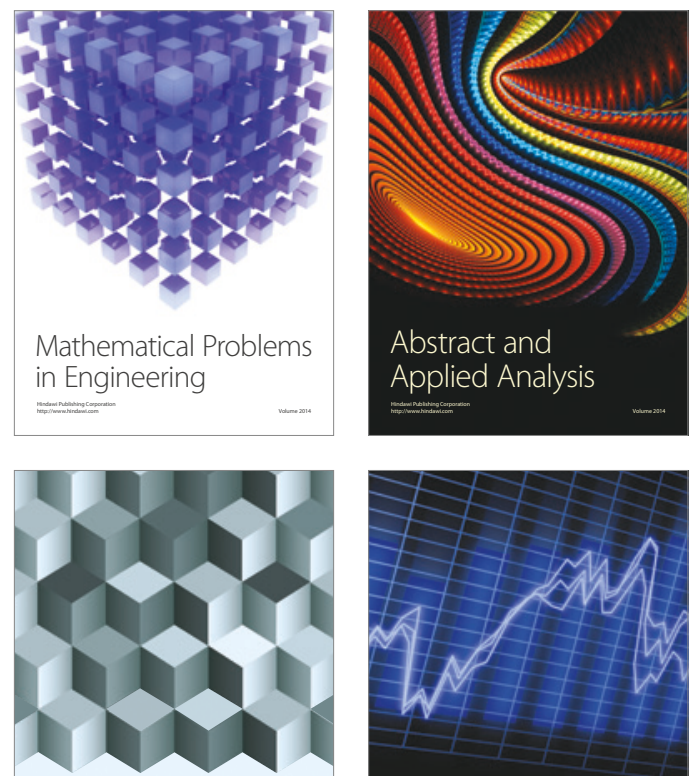

Journal of

Function Spaces

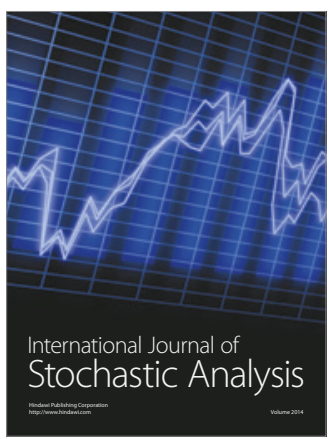

Probability and Statistics
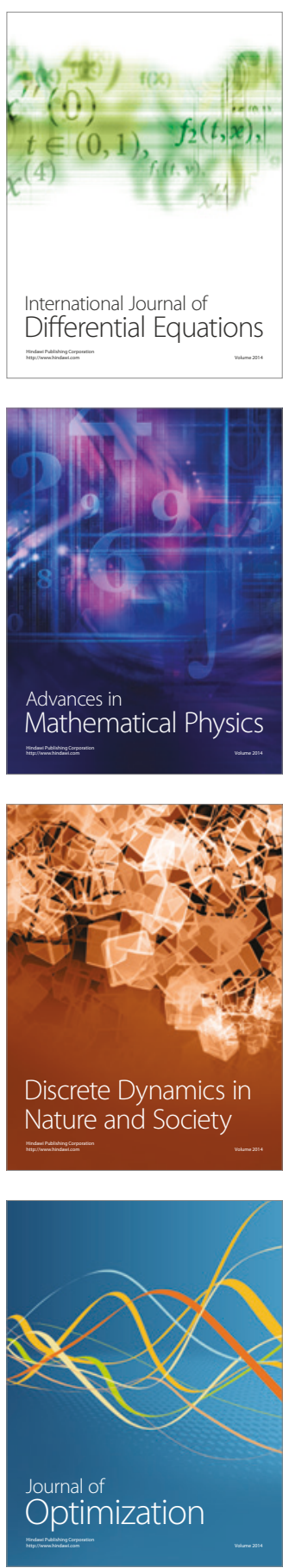